\title{
Parrot Beak Nails Revisited: Case Series and Comprehensive Review
}

Stella X. Chen · Philip R. Cohen

Received: November 21, 2017 / Published online: December 19, 2017

(C) The Author(s) 2017. This article is an open access publication

\section{ABSTRACT}

The term "parrot beak nail" describes a morphologic change of the nail plate characterized by excessive forward curvature. It may be associated with systemic disease or, most commonly, occurs as an idiopathic finding complicated by delayed nail plate trimming. The characteristics of parrot beak nails in ten men are described, and the features of this acquired nail deformity are reviewed. Of the ten patients, six presented with concurrent neuropathies that resulted in frequent foot injuries or falls. While the true incidence of parrot beak nails is unknown, this nail deformity occurred in $2.1 \%$ of patients seen by a single physician during a 3-month period. In conclusion, parrot beak nails secondary to poor nail care may lead to functional impairment, tissue injury, and subsequent infections. Therefore, it is important for clinicians to look for these nail lesions on cutaneous examination and

Enhanced content To view enhanced content for this article go to http://www.medengine.com/Redeem/ 9CFCF0602C646590.

S. X. Chen $(\bowtie)$

School of Medicine, University of California San

Diego, La Jolla, CA, USA

e-mail: sxchen@ucsd.edu

P. R. Cohen $(\varangle)$

Department of Dermatology, University of

California San Diego, La Jolla, CA, USA

e-mail: mitehead@gmail.com recommend frequent nail trimmings to individuals with parrot beak nails.

Keywords: Fingernails; Nails; Nail bed; Nail plate; Neuropathy; Parrot beak; Parrot beak nails

\section{INTRODUCTION}

Parrot beak nails are benign nail lesions characterized by excessive forward curvature of the free nail edge, resulting in the eponymous resemblance to a parrot's beak. It is most commonly an idiopathic finding, although it may also occur secondary to systemic processes. Over a 3-month period, ten men with parrot beak nails were diagnosed. The characteristics of these individuals are described, and the features of this acquired nail deformity are reviewed.

\section{CASE SERIES}

Ten men with parrot beak nails were diagnosed during a 3-month period, representing $2.1 \%$ of patients seen by one dermatologist. This period included a total of 303 men and 133 women; that all patients diagnosed with parrot beak nails were male is likely related to the increased distribution of men seen in the clinic (69\% men, $31 \%$ women). The ages, locations of 
involvement, associated pedal findings, and associated systemic disorders of these patients are summarized in Table 1.

Parrot beak nails were observed in ten men between the ages of 63 and 89 years; the median age was 74 years. The diagnosis was made clinically based on the characteristic curvature of the free nail edge (Fig. 1). All of the men had toenail involvement; most presented with multiple affected nails (Fig. 2). None, however, demonstrated fingernail involvement. The nail edge extended directly into the soft tissue of the digit pad, leading to pain or impairment of ambulation for some of the patients (Fig. 3). However, for several of the men, the parrot beak nails were an incidental finding seen on examination.

In addition to parrot beak nails, additional nail conditions and toe abnormalities were noted (Fig. 4). Two men presented with subungual hematomas (cases 3 and 10), two men had

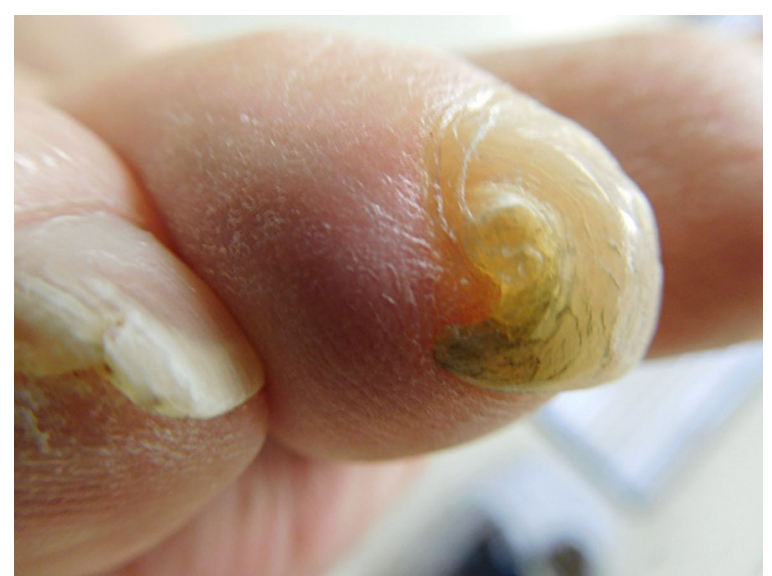

Fig. 1 Forward curvature of a parrot beak nail seen on the right third toe of a 75-year-old Caucasian man (case 6)

hammer toes (cases 3 and 6), and two patients exhibited onychodystrophy (cases 2 and 5).

Distal neuropathy was present in six of the ten patients (Table 1). One man, demonstrating

Table 1 Clinical characteristics of ten Caucasian men with parrot beak nails

\begin{tabular}{lllll}
\hline C & $\begin{array}{l}\text { Age } \\
\text { (years) }\end{array}$ & $\begin{array}{c}\text { Toenails } \\
\text { involved }\end{array}$ & Associated pedal findings & Systemic disorders \\
\hline 1 & 63 & R5 & Onycholysis (L1) & Pedal neuropathy due to spinal stenosis \\
2 & 64 & $\begin{array}{c}\text { R2-R5, } \\
\text { L3-L5 }\end{array}$ & Onychogryphosis (L1, L2) & $\begin{array}{c}\text { Diabetic neuropathy, history of cardiac } \\
\text { transplant, chronic kidney disease }\end{array}$ \\
3 & 68 & R2-R4 & $\begin{array}{c}\text { Subungual hematoma (R3), hammer toe } \\
\text { (R2), longitudinal erythronychia (L1) }\end{array}$ & $\begin{array}{c}\text { Distal neuropathy secondary to androgen } \\
\text { deprivation therapy for prostate cancer }\end{array}$ \\
4 & 70 & L2 & None & None \\
5 & 72 & L2 & Onychauxis (L1) & None \\
6 & 75 & R3, L3 & Hammer toe (L3) & Multiple system atrophy \\
7 & 76 & R2, R3 & None & None \\
8 & 80 & L2-L4 & Overlying left fifth toe & Coronary artery disease \\
9 & 88 & L2-L5 & None & Pedal neuropathy \\
10 & 89 & R2-R4, & Subungual hematoma (R2) & Pedal neuropathy, bladder cancer, prostate \\
& & L2-L4 & & $\begin{array}{c}\text { cancer, lymphoplasmacytic sclerosing } \\
\text { pancreatitis }\end{array}$ \\
\hline
\end{tabular}

$C$ case, $L$ left, $R$ right, 2 second toe, 3 third toe, 4 fourth toe, 5 fifth toe

${ }^{a}$ Multiple system atrophy is a neurodegenerative disease consisting of autonomic, cerebellar, and pyramidal dysfunction with parkinsonian features [10]

${ }^{b}$ Lymphoplasmacytic sclerosing pancreatitis is an inflammatory pancreatic disorder characterized by autoimmune destruction and fibrosis of the pancreas [11] 

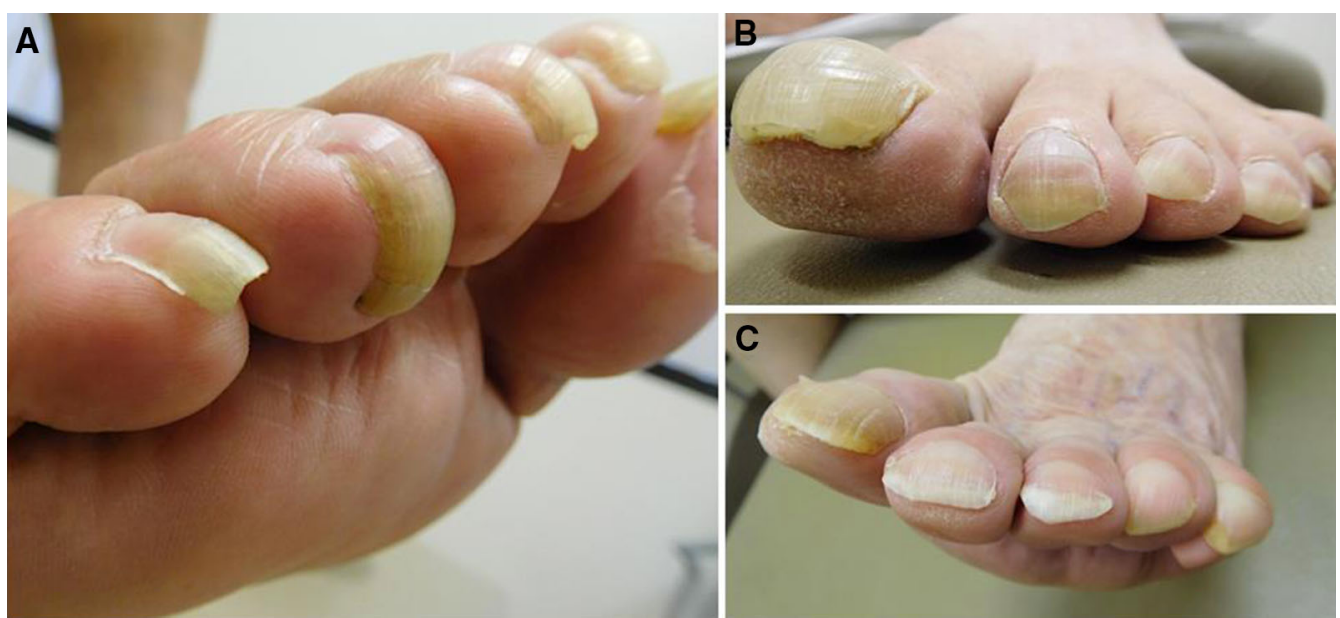

Fig. 2 Parrot beak nails involving the right second through fifth toes (a) of a 64-year-old man (case 2), the left second toe (b) of a 72-year-old man (case 5), and the left second through fifth toes (c) of an 88-year-old man (case 9)
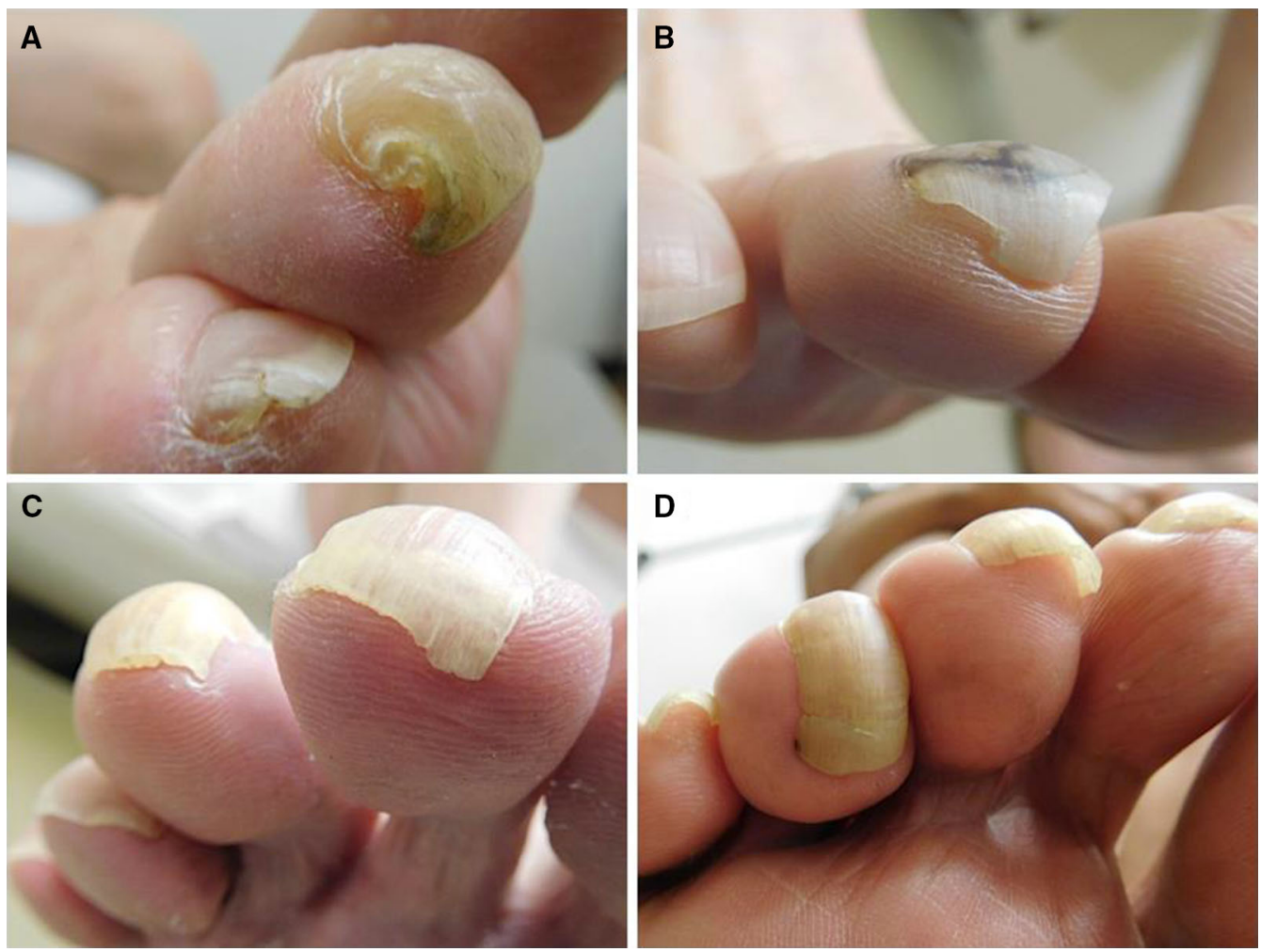

Fig. 3 Parrot beak nails that pierce the distal toe. The nail of the right third toe of a 75 -year-old man with multiple system atrophy (case 6) pierces the tissue (a). The right third toenail of a 68 -year-old patient with a subungual hematoma (case 3) presses into the distal toe $(\mathbf{b})$. The nails

of the right second and third toes of a 76-year-old man (case 7) extend into the tissue of the digits (c). The right fourth toenail of a 64-year-old man (case 2) pushes against the distal toe $(\mathbf{d})$ 

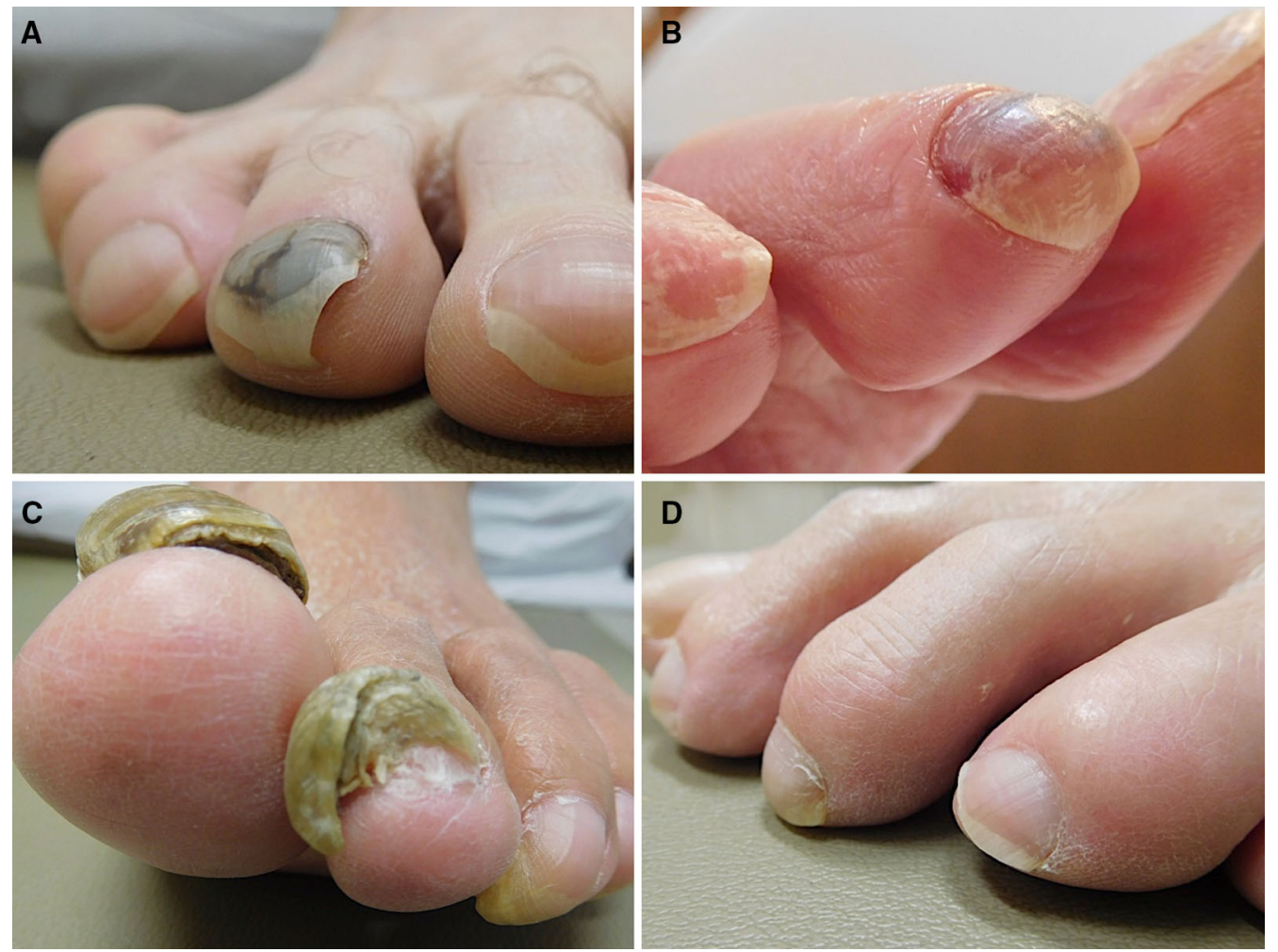

Fig. 4 Concurrent nail deformities can be observed in patients with parrot beak nails. Subungual hematoma (a) beneath the right third toenail of a 68-year-old man with neuropathy secondary to androgen deprivation therapy secondary to prostate cancer (case 3). Subungual hematoma (b) beneath the right second toenail of an

89-year-old man (case 10). Onychodystrophy of the first and second left toes alongside a parrot beak nail of the left third toenail (c) of a 64-year-old man with diabetic neuropathy (case 2). Hammer toe (d) of the left third toe of a 75-year-old man with multiple system atrophy (case 6)

a parrot beak nail on the right fifth toenail, had longstanding neuropathy due to spinal stenosis (case 1). Another man with involvement of seven toenails had history of severe diabetic neuropathy (case 2). A third man developed distal neuropathy as a sequela to androgen deprivation therapy started for prostate cancer (case 3). Two other men reported idiopathic peripheral neuropathy (cases 9 and 10). While these five men all reported lower extremity sensory neuropathy, a sixth man with intact sensation-but cerebellar and motor dysfunction secondary to multiple system atrophyalso presented with parrot beak nails (case 6). This patient presented with a hammer toe on examination, and had history of frequent falls

and foot trauma. Other systemic disorders in the patients with parrot beak nails are listed in Table 1.

All of the patients were uncertain of the onset of their nail deformities. Most reported infrequent clipping of the nails. One man commented that he had not cut his nails for at least 1 year (case 2).

The patients were advised to soak their nails in water before cutting the free forward edge. Two men returned to our clinic and demonstrated successful removal of the edges of their parrot beak nails. They also had concurrent symptomatic relief (cases 3 and 9, Fig. 5).

Informed consent was obtained from all patients for being included in the study. 

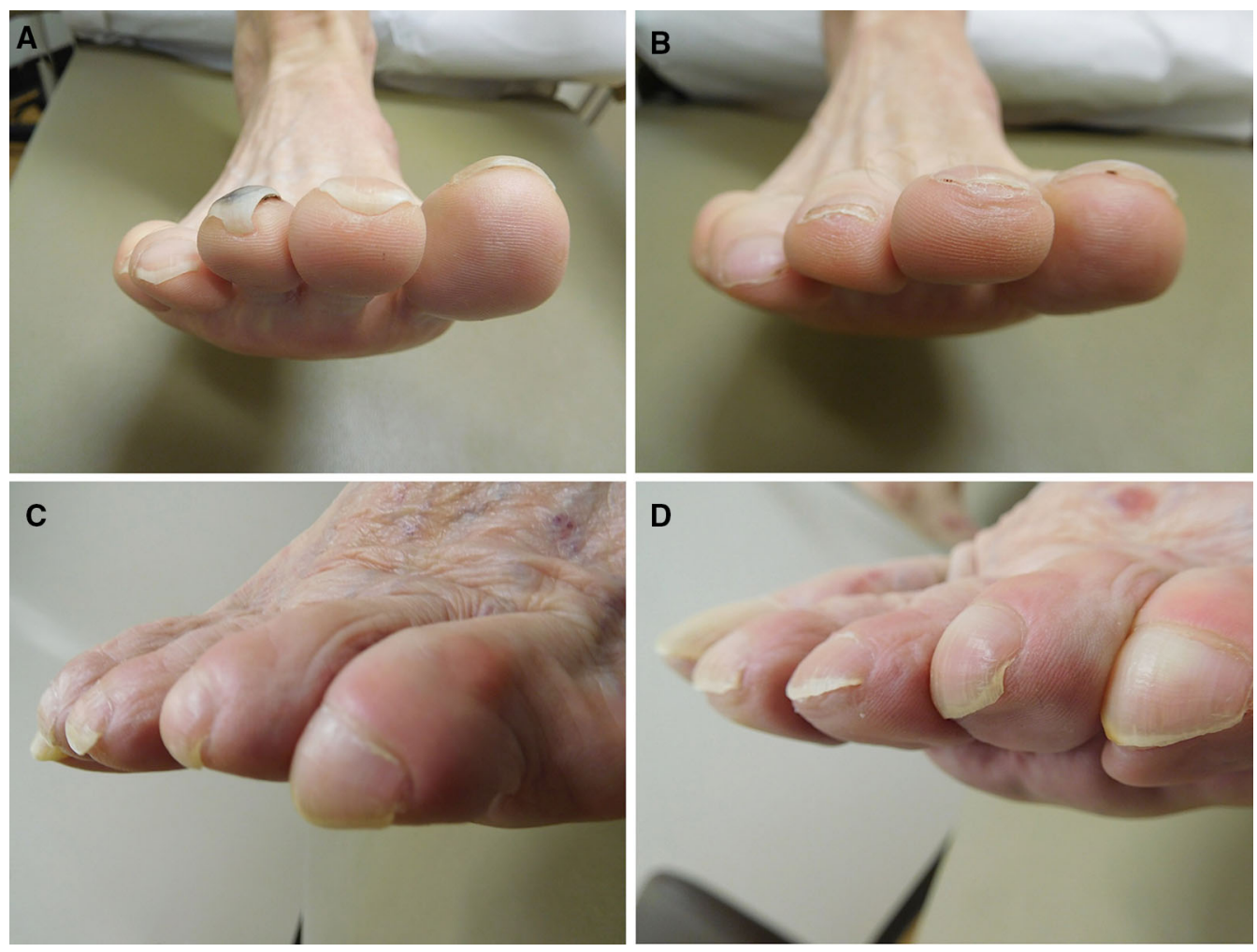

Fig. 5 Resolution of parrot beak nails after toenail clipping. Parrot beak nails of the right second, third, and fourth toes and subungual hematoma of the right third toenail (a) of a 68-year-old man before toenail trimming (case 3). Improvement of parrot beak nails and subungual hematoma on these toes after trimming; there is residual downward curving of the nail plate (b). Parrot beak nails

\section{DISCUSSION}

Parrot beak nails are a benign deformity of the nail plate characterized by excessive, symmetric forward curvature. The term was first coined by Kandil in 1971, who reported a peculiar, idiopathic overcurvature of the third and fourth free fingernail edges in a 38-year-old woman [1]. Since then, idiopathic parrot beak nails have been described in three additional women, as presented in Table 2 [1-5].

In 1993, Kurokawa et al. described parrot beak toenail deformities in two girls: the right third and fourth toes of an 11-year-old girl and the right fourth toe of a 17-year-old girl. Both of the girls also had congenital anomalies such as soft tissue or bony hypoplasia in the adjacent or

of the left second, third, fourth, and fifth toes (c) of an 88-year-old man (case 9). Improvement of parrot beak nails on these toes following toenail clipping, with residual downward curvature most prominent in the left fourth toenail $(\mathbf{d})$

involved toes. Specifically, the 11-year-old girl had congenital absence of soft tissues in the toe tips of her right second toe and left fourth toe, whereas the 17-year-old girl had severe bony hypoplasia of the distal phalanx of her right fourth toe [2].

More recently, Desai et al. also reported idiopathic (or primary) parrot beak nails affecting all of the fingernails of a 55-year-old woman who was otherwise healthy [3]. Parrot beak nails have also been shown to occur secondary to conditions causing finger pulp atrophy [3]. Secondary parrot beak nails have been associated with chronic crack cocaine use and as one of many possible nail lesions observed in patients with systemic sclerosis $[4,5]$.

Prior to our series, all individuals with idiopathic parrot beak nails have been women 
Table 2 Characteristics of patients with parrot beak nails published in literature

\begin{tabular}{|c|c|c|c|c|c|c|}
\hline $\begin{array}{l}\text { Author } \\
\text { Year }\end{array}$ & Ref. & Study type & Gender & Age (years) & Location & Associations \\
\hline $\begin{array}{l}\text { Kandil } \\
1971\end{array}$ & {$[1]$} & Case report $(n=1)^{a}$ & Female & 38 & $\begin{array}{l}\text { Third and fourth } \\
\text { fingernails }\end{array}$ & Idiopathic \\
\hline $\begin{array}{l}\text { Kurokawa } \\
\text { et al. } \\
1993\end{array}$ & [2] & Case series $(n=2)^{\mathrm{b}}$ & Female & $\begin{array}{l}11-17 \\
\quad(m=14)\end{array}$ & Toenails & $\begin{array}{l}\text { Congenital soft tissue and bony } \\
\text { abnormalities } \\
\text { Idiopathic }\end{array}$ \\
\hline $\begin{array}{l}\text { Payne-James } \\
\text { et al. } \\
2007\end{array}$ & {$[4]$} & Case series $(n=8)^{c}$ & Female & $\begin{array}{l}24-40 \\
\quad(m=30)\end{array}$ & Fingernails & Chronic crack cocaine use \\
\hline $\begin{array}{l}\text { Desai et al. } \\
2011\end{array}$ & [3] & Case report $(n=1)^{\mathrm{d}}$ & Female & 55 & All fingernails & Idiopathic \\
\hline $\begin{array}{l}\text { Marie et al. } \\
2017\end{array}$ & {$[5]$} & $\begin{array}{l}\text { Case-control study } \\
\quad(n=42)^{\mathrm{e}}\end{array}$ & $\begin{array}{l}\text { Not } \\
\text { stated }\end{array}$ & Not stated & Fingernails & $\begin{array}{l}\text { Idiopathic } \\
\text { Systemic sclerosis }\end{array}$ \\
\hline $\begin{array}{c}\text { Chen and } \\
\text { Cohen }\end{array}$ & $\mathrm{CR}$ & Case series $(n=10)^{\mathrm{f}}$ & Male & $\begin{array}{l}63-89 \\
\quad(m=74)\end{array}$ & Toenails & $\begin{array}{l}\text { Idiopathic } \\
\text { Pedal findings }\end{array}$ \\
\hline 2017 & & & & & & Systemic disorders \\
\hline
\end{tabular}

$C R$ current report, $M$ median, $N$ number of patients, Ref reference

${ }^{a}$ A 38-year-old housewife dated her condition to the time when she stopped washing utensils; she developed symmetrical bilateral overcurvature of the free margins of the third and fourth fingernails. The overcurvature disappeared temporarily after soaking the nails in tepid water for $30 \mathrm{~min}$

b Two patients were diagnosed with idiopathic parrot beak nails involving the right third and fourth toes of an 11-year-old girl and the right fourth toe of a 17-year-old girl. Both were also noted to have congenital soft tissue or bony hypoplasia in the adjacent or involved toes: the right second toe of an 11-year-old girl (adjacent to parrot beak nail), and the right fourth toe (involved toe of parrot beak nail) of a 17-year-old girl

${ }^{c}$ Parrot beak nails were reported in the fingernails of eight women with chronic crack cocaine use in the UK. These findings were part of a "pseudoscleradermatous triad" consisting of perniosis, pulp atrophy, and parrot beak nails

d A 55-year-old healthy woman reported 5-year history of parrot beak nails involving all fingernails. Symmetrical free margin overcurvature temporarily straightened after a warm bath. When permitted to grow long, the nails led to functional difficulties

e 129 patients with systemic sclerosis and 80 healthy controls were evaluated for fingernail changes in a case-control study. The study showed parrot beak fingernail changes not only in 40 of the patients with systemic sclerosis, but also in 2 of the healthy controls. The gender was not specified for these individuals. The incidence of parrot beak nails was $31 \%$ in patients with systemic sclerosis and $2.5 \%$ in healthy individuals

${ }^{f}$ Ten Caucasian men seen at a single institution were diagnosed with idiopathic parrot beak nails involving the toenails. The following pedal findings were also observed: hammer toe ( 2 patients), longitudinal erythronychia ( 1 patient), onychauxis (1 patient), onychogryphosis ( 1 patient), onycholysis ( 1 patient), overlying toe ( 1 patient), and subungual hematoma ( 2 patients). In addition, the following systemic disorders were observed: bladder cancer ( 1 patient); chronic kidney disease (1 patient); coronary artery disease (1 patient); history of cardiac transplant (1 patient); lower extremity neuropathies including diabetic neuropathy (1 patient), multiple system atrophy (1 patient), and pedal neuropathy (4 patients); lymphoplasmacytic sclerosing pancreatitis (1 patient); prostate cancer (1 patient) 
(Table 2). Including this report, 64 individuals with parrot beak nails have been reported. The youngest patient was an 11-year-old girl, while the oldest patient was an 89-year-old man (case 10, Table 1).

Parrot beak nails were seen in $2.5 \%$ of healthy patients in a single center [5]. In our institution, this nail abnormality was diagnosed in $2.1 \%$ of patients seen within a 3-month period by a single dermatologist. All individuals with parrot beak nails at our institution were male, which likely reflects the increased number of male patients seen (303 men as compared with 133 women seen during the 3-month period). Parrot beak nails are usually asymptomatic and are typically noticed as incidental findings; therefore, the actual incidence of parrot beak nails is likely to be higher.

The clinical presentation of the parrot beak nail involves the free edge of the nail symmetrically curved in an elliptical shape over the distal tip of the finger, similar to the curvature seen in a parrot's beak. The integrity of the overlying nail plate is unaffected and curves in a normal, circular fashion; consequently, parrot beak nails may not be seen until the nails are permitted to grow for some time [1]. The diagnosis of parrot beak nails is typically made clinically based on the characteristic curvature of the free nail edge. However, a more specific diagnostic test involves soaking the nail in warm water for $30 \mathrm{~min}$, after which the nail plate will temporarily straighten out $[1,3]$.

Parrot beak nails may affect fingernails or toenails, and can affect a single or multiple nails. In our series, a median of three toenails was affected in ten patients. Eight of ten patients had three or fewer toenails involved; three patients had isolated involvement of one toenail. A maximum of four toenails were affected on one foot in a single patient; he had involvement of seven toenails between both feet (case 2, Table 1). Involvement may be unilateral (seven men, Table 1) or bilateral (three men, Table 1). None of our patients had involvement of their great toenails.

The nail plate, nail bed, and surrounding soft tissue are morphologically normal and painless. However, pain, tissue damage, and functional difficulties may occur if the nail is permitted to grow into the digital tissue. While usually asymptomatic, parrot beak nails may be cosmetically distressing to the patient and invoke anxiety.

Parrot beak nails are characterized by abnormal forward curvature at the tip of an unaffected nail bed and nail plate. This otherwise ordinary nail phenotype distinguishes it from other nail disorders; For instance, the normal curvature of the attached nail plate differentiates parrot beak nails from clubbing, another nail curvature abnormality associated with impaired pulmonary perfusion. The differential diagnosis of parrot beak nails also includes the curved nails seen in pachyonychia congenita; however, parrot beak nails are not associated with discolored or thickened nails. Other nail curvature abnormalities include claw-like nails secondary to ill-fitting shoes, congenital curvature of the fourth toenail, distal hemitorsion in porphyria, and Kirner's deformity [6].

The etiology of parrot beak nails is currently unknown. They were first reported as an idiopathic finding in an otherwise healthy individual, but may also occur congenitally, possibly secondary to an underlying skeletal or soft tissue abnormality $[1,2]$. Parrot beak nails have also been characterized as part of a triad associated with chronic crack cocaine abuse [4]. The concurrent presence of parrot beak nails, perniosis, and finger pulp atrophy should alert the clinician to consider the possibility of chronic crack cocaine use. The nail changes occur as a result of vasoconstrictive ischemia of the peripheral digits [4]. More recently, parrot beak nails were characterized as one of many nail lesions seen in systemic sclerosis, being more commonly seen after 3 years of disease [5].

Repeated trauma to the nail bed is another potential cause for the development of parrot beak nails. The parrot beak nails of the men seen at our institution developed idiopathically. However, six of the ten patients reported distal neuropathies: five individuals had sensory deficits of the digits (cases 1-3, 9, 10), and one man had motor neuropathy secondary to multiple system atrophy (case 6).

Although the neuropathy is unlikely to directly cause the parrot beak nails, the clinical 
consequence of this impairment may result in gait abnormalities or repeated foot trauma that occur as a result of impaired sensorium, leading to injuries within the growing nail bed and subsequent parrot beak nail development. The individual with multiple system atrophy did not report sensory neuropathy, but experienced frequent falls and foot trauma as a result of motor weakness. He presented with a hammer toe, an example of a pedal abnormality associated with repeated foot trauma or gait abnormalities, in addition to parrot beak nails. The other reports of parrot beak nails do not state whether peripheral neuropathy was present; therefore, it is possible that this feature may have been underreported.

The pathogenesis of parrot beak nails remains to be established. The nail plate has three zones: the physically hard dorsal nail plate and thin (one to two cells in thickness) ventral nail plate have higher phospholipid, calcium, and sulfhydryl group contents in comparison with the encompassed, intermediate nail plate, which has higher acid phosphatase activity [7]. Since the abnormal curvature may be rescued following water submersion, the etiology of idiopathic parrot beak nails may involve aberrances in hydrophobic phospholipid content in one of the three nail plate zones.

An injury sustained to the growth region of the nail bed may traumatize any of the three developing nail plate zones, ultimately resulting in an imbalance of growth or hydrophobic content among the zones and exaggerated longitudinal curvature. Thus, idiopathic parrot beak nails may develop as a result of repeated trauma to the nail matrix. In our series, six men who presented with parrot beak nails also had history of frequent foot injuries (cases 1-3, 6, 9, 10).

In addition, overt digital amputation or tight surgical closure during fingertip procedures may lead to finger pulp atrophy and extensive fingertip scarring, causing a hooked-nail deformity closely resembling parrot beak nails [8]. This consequence of orthopedic trauma and surgery occurs after the loss of nail support from the terminal phalanx complicated by extensive scarring [9]. Several surgical techniques have been shown to prevent this parrot beak-like deformity following fingertip injuries, including nail relocation and the use of hypodermic needles for tension-free closure $[8,9]$.

Other mechanisms for the development of parrot beak nails have been postulated. Congenital parrot beak nails may develop due to underlying or adjacent bone or soft tissue abnormalities of the involved or adjacent toe [2]. Parrot beak nails may also develop as a sequela of peripheral ischemia in amphetamine-induced or systemic sclerosis-associated peripheral vasoconstriction [4].

Patients with parrot beak nails should be advised to trim their nails frequently. If the nails have grown long enough that nail-clipping becomes difficult, patients may soak their nails in water-such as during bathing or showering-for at least $5 \mathrm{~min}$ (or up to $30 \mathrm{~min}$ )- to allow for straightening and softening of the nail before clipping [1]. This was recommended to our patients; two men returned to the office with clipped nails and symptomatic relief, one of whom also saw resolution of a prior subungual hematoma (Fig. 5).

However, in our patients, nail clipping did not cure the parrot beak nail deformity; the free edge of the nail plate continues to subtly curve downwards (Fig. 5). Nevertheless, it is important to keep parrot beak nails trimmed to prevent growth of the nail into soft tissue. This is especially critical for patients who may have underlying impaired wound healing, such as those with chronic venous stasis, diabetes, immunosuppression, and peripheral vascular disease.

\section{CONCLUSIONS}

The term "parrot beak nail" describes a morphologic change of the nail plate characterized by excessive forward curvature appearing similar to the beak of a parrot. It is typically an incidental finding; however, it can also occur congenitally in patients with skeletal abnormalities or associated with either systemic sclerosis or chronic crack cocaine use. Ten men $(2.1 \%$ of patients seen in our office during a 3-month period) were diagnosed with parrot beak nails. All of the men developed the nail changes idiopathically, although six patients also reported distal neuropathies that led to frequent foot and toe injuries. The pathogenesis 
of parrot beak nails may involve ischemia during vasoconstrictive processes such as scleroderma or amphetamine use; yet, we also postulate that frequent injuries to the nail bed from digit trauma may cause disruption to nail plate zones, leading to exaggerated longitudinal curvature. Patients with parrot beak nails may experience soft tissue damage or functional impairment if the nails are permitted to grow. Thus, it is important for patients with parrot beak nails to frequently trim their nails, especially in those who are susceptible to infection and chronic wounds.

\section{ACKNOWLEDGEMENTS}

Funding. No funding or sponsorship was received for this study or the publication of this article.

Authorship. All named authors meet the International Committee of Medical Journal Editors (ICMJE) criteria for authorship for this manuscript, take responsibility for the integrity of the work as a whole, and have given final approval of the version to be published.

Disclosures. The authors, Stella X. Chen, BS, and Philip R. Cohen, MD, have nothing to disclose.

Compliance with Ethics Guidelines. Informed consent was obtained from all patients for being included in the study.

Open Access. This article is distributed under the terms of the Creative Commons Attribution-NonCommercial 4.0 International License (http://creativecommons.org/licenses/ by-nc/4.0/), which permits any noncommercial use, distribution, and reproduction in any medium, provided you give appropriate credit to the original author(s) and the source, provide a link to the Creative Commons license, and indicate if changes were made.

\section{REFERENCES}

1. Kandel E. Parrot beak nails. J Med Liban. 1971;244:433-436. https://www.ncbi.nlm.nih.gov/ pubmed/5146639. Accessed 21 Nov 2017

2. Kurokawa M, Isshiki N, Inoue K. A new treatment for parrot beak deformity of the toe. Plast Reconstr Surg. 1994;93:558-560. https://www.ncbi.nlm.nih. gov/pubmed/8115511. Accessed 21 Nov 2017

3. Desai T, Magdum A, Patel T, Loghdey S. Parrot-beak nails. Clin Exp Dermatol. 2011;36:208-9. https:// doi.org/10.1111/j.1365-2230.2010.03899.x.

4. Payne-James JJ, Munro MH, Rowland Payne CM. Pseudosclerodermatous triad of perniosis, pulp atrophy and 'parrot-beaked' clawing of the nails-a newly recognized syndrome of chronic crack cocaine use. J Forensic Leg Med. 2007;14:65-71. https://www.ncbi.nlm.nih.gov/pubmed/17650550. Accessed 21 Nov 2017

5. Marie I, Gremain V, Nassermadji K, Richard L, Joly $P$, Menard JF, Levesque $H$. Nail involvement in systemic sclerosis. J Am Acad Dermatol. 2017;76:1115-23. https://doi.org/10.1016/j.jaad. 2016.11.024.

6. Rubin AI, Baran R. Physical signs: Baran and Dawber's diseases of the nails and their management. Hoboken: Wiley; 2012. p. 51-99.

7. de Berker DA, Baran R. Science of the nail apparatus: Baran and Dawber's diseases of the nails and their management. Hoboken: Wiley; 2012. p. 1-50.

8. Pandya AN, Giele HP. Prevention of the parrot beak deformity in fingertip injuries. Hand Surg. 2001;6:163-166. https://www.ncbi.nlm.nih.gov/ pubmed/11901462. Accessed 21 Nov 2017

9. Krishna BV, Pelly AD. Nail relocation by nail flap in digital injuries. Br J Plast Surg. 1982;35:53-57. https://www.ncbi.nlm.nih.gov/pubmed/7066587.

10. Fanciulli A, Wenning GK. Multiple system atrophy. N Engl J Med. 2015;372:249-63. https://doi.org/10. 1056/NEJMra1311488.

11. Kram MT, May LD, Cooperman A, Bernstein S, Abedeer R, Cohen PR. Lymphoplasmacytic sclerosing pancreatitis and cholangitis. Gastrointest Endosc. 2002;55:588-90. https://www.ncbi.nlm. nih.gov/pubmed/11923781. 